\title{
Moutan Cortex Extract Exerts Protective Effects in a Rat Model of Cardiac Ischemia/Reperfusion
}

\begin{tabular}{|c|c|}
\hline Journal: & Canadian Journal of Physiology and Pharmacology \\
\hline Manuscript ID & cjpp-2015-0168.R2 \\
\hline Manuscript Type: & Article \\
\hline Date Submitted by the Author: & 17-Jul-2015 \\
\hline Complete List of Authors: & $\begin{array}{l}\text { Dan, Hong; Key Laboratory of Chinese Medicine Resource and Compound } \\
\text { Prescription (Hubei University of Chinese Medicine), Ministry of Education, } \\
\text { Zhang, Liping; Key Laboratory of Chinese Medicine Resource and } \\
\text { Compound Prescription (Hubei University of Chinese Medicine), Ministry of } \\
\text { Education, } \\
\text { Qin, Xiaolin; Key Laboratory of Chinese Medicine Resource and Compound } \\
\text { Prescription (Hubei University of Chinese Medicine), Ministry of Education, } \\
\text { Peng, Xiaohui; Key Laboratory of Chinese Medicine Resource and } \\
\text { Compound Prescription (Hubei University of Chinese Medicine), Ministry of } \\
\text { Education, } \\
\text { Wong, Mingyan; Key Laboratory of Chinese Medicine Resource and } \\
\text { Compound Prescription (Hubei University of Chinese Medicine), Ministry of } \\
\text { Education, } \\
\text { Tan, Xuan; Union Hospital, Tongji Medical College, Huazhong University of } \\
\text { Science and Technology, } \\
\text { Yu, Shanggong; Arkansas Children's Nutrition Center, } \\
\text { Fang, Nianbai; Arkansas Children's Nutrition Center, }\end{array}$ \\
\hline Keyword: & $\begin{array}{l}\text { Moutan Cortex, Ischemia/Reperfusion, cardioprotective, anti-oxidative, } \\
\text { anti-apoptotic }\end{array}$ \\
\hline
\end{tabular}

\section{SCHOLARONE" \\ Manuscripts}


3 Moutan Cortex Extract Exerts Protective Effects in a Rat Model of

$9{ }^{1}$ Key Laboratory of Chinese Medicine Resource and Compound Prescription (Hubei 10 University of Chinese Medicine), Ministry of Education, 1 Huang-jia-hu, Wuhan, 11 China; ${ }^{2}$ Union Hospital, Tongji Medical College, Huazhong University of Science and

12 Technology, 1277 Jiefang Avenue, Wuhan, China; ${ }^{3}$ Arkansas Children's Nutrition Center,

13 Little Rock, AR 72202; ${ }^{4}$ Departments of Pediatrics, University of Arkansas for Medical

14 Sciences, Little Rock, AR 72205

15

16 * Corresponding author. E-mail:NFang@uams.edu (N.F.);

17 Telephone number: 501-364-2747;

18 Fax number: 501-364-3161 
1

2

3 The present study was performed to investigate the effects of MC on myocardial

4 ischemia/reperfusion (I/R) in rats and to explore its possible mechanisms. Sprague-

5 Dawley rats were administered MC extract (1.98 g/kg, i.g.) for 14 days and underwent a

6 subsequent open-chest procedure involving $30 \mathrm{~min}$ of myocardial ischemia and $60 \mathrm{~min}$

7 of reperfusion. The cardioprotective effect of $\mathrm{MC}$ was demonstrated by reduced infarct

8 size and marked improvement in the histopathological examination. The increase in the

9 activity of superoxide dismutase (SOD) and glutathione (GSH) as well as the reduction

10 of malondialdehyde (MDA) indicated that MC effectively promoted the anti-oxidative

11 defense system. Increased anti-oxidative defense was accompanied by decreased

12 release of lactate dehydrogenase $(\mathrm{LDH})$ and creatine kinase $(\mathrm{CK})$. The reduction in

13 TUNEL-positive myocytes demonstrated that MC decreased myocardial apoptosis. The

14 mRNA expression of B-cell leukemia-2 (Bcl-2) was up-regulated by $\mathrm{MC}$ and the ratio of

15 Bcl-2/ Bcl-2-associated $X$ protein (Bax) mRNA expression was increased. MC 16 pretreatment decreased the mRNA expression of inducible nitric oxide synthase (iNOS).

17 The data from this study suggest that MC exerted protective effects on acute myocardial $18 \quad$ I/R injury via anti-oxidative and anti-apoptotic activities. apoptotic 
1

\section{Introduction}

The World Health Organization estimates that heart failure initiated by coronary heart disease and myocardial infarction is one of the leading causes of death worldwide (Venugopal et al. 2012). In acute myocardial infarction, coronary reperfusion using percutaneous coronary intervention $(\mathrm{PCl})$ or thrombolysis is the standard therapy for salvaging the myocardium and reducing the onset of heart failure (Gershlick et al. 2013). However, reperfusion itself may lead to accelerated or additional damage, also known as myocardial ischemia/reperfusion (I/R) injury (Dominguez-Rodriguez et al. 2014).No effective therapy is currently available to mitigate the consequences of $I / R$ (Pagliaro and Penna 2014).

Moutan Cortex (MC), the root cortex of Paeonia suffruticosa Andrews, has been commonly used in traditional Chinese medicine to promote blood circulation and to alleviate blood stasis (Chen et al. 2005). MC extract was reported to exhibit a variety of biological activities, including anti-inflammatory (Fu et al. 2012; Wu and Gu 2009; Yun et al. 2013), anti-allergic (Jiang et al. 2007; Liu et al. 2013), and anti-oxidative effects (Rho et al. 2005; Zhang et al. 2014). Previous studies concerning its anti-oxidative activity indicated that MC extract attenuated oxidative stress in AGE-induced mesangial cell dysfunction and in streptozotocin-induced diabetic nephropathy (Zhang et al. 2014). MC extract also inhibited reactive oxygen species (ROS) production in oxidativestressed PC12 cells (Rho et al. 2005). Oxidative stress due to ROS is thought to play an important role in I/R injury that impairs cardiac function (Inafuku et al. 2013). ROS are produced in large quantities in the first few minutes of $\mathrm{I} / \mathrm{R}$ (Dominguez-Rodriguez and 
1 Abreu-Gonzalez 2010). Many herbal antioxidants have been tested for the treatment of

$2 \mathrm{I} / \mathrm{R}$ injury. Whether the administration of $M C$, known to possess antioxidant activity,

3 would reduce oxidative stress induced by $\mathrm{I} / \mathrm{R}$ has not been investigated.

$4 \quad$ I/R injury are a complex set of events that paradoxically causes tissue injury. There

5 is now growing evidence suggesting a close link between oxidative stress and the

6 induction of apoptosis in the process of I/R (Dobsak et al. 2003). Various studies have

7 demonstrated that both ROS and its oxidation products and other secondary messenger

8 molecules generated by ROS can trigger programmed cell death (Mohanty et al. 2006).

9 In the present experiment, we used an in vivo rat model to study whether MC can

10 attenuate heart injury induced by $\mathrm{I} / \mathrm{R}$ and the role of oxidative stress and apoptosis in

11 this process.

12 Materials and methods

13 Plant material

14 The plant samples were collected from the tree peony (Paeonia suffruticosa

15 Andrews) grown in the Fenghuangshan area in Anhui Province. The samples were

16 kindly provided by Prof. Qinglin Li from Anhui University of Chinese Medicine and were

17 identified by Prof. Keli Chen from the Department of Pharmacognosy, Hubei University

18 of Chinese Medicine. A voucher specimen was preserved in the Key Laboratory of

19 Chinese Medicine Resource and Compound Prescription (Hubei University of Chinese

20 Medicine), Ministry of Education, Wuhan, China.

21 The MC extract 
1

2

4 stored at $-20^{\circ} \mathrm{C}$ until use. The constituents in $\mathrm{MC}$ were analyzed by liquid

5 chromatography-tandem mass spectrometry (LC-MS/MS) and high-performance liquid

6 chromatography (HPLC) (see Supplementary material for details).

\section{Animals}

8 Male Wistar rats (200-250 g) were purchased from Hubei Provincial Center for

9 Disease Control and Prevention, China (certificate No.: SCXK 2008-0005). Animals 10 were maintained in a temperature-controlled room $\left(22 \pm 2^{\circ} \mathrm{C}\right)$ and kept on a 12-h

11 light/dark cycle. Food and water were available ad libitum. All rats were allowed to

12 acclimate for a week before testing. Animal care and treatment were approved prior to 13 the study by the Animal Care Committee of Hubei University of Chinese Medicine, 14 Hubei, China.

\section{Surgical procedure}

16 Myocardial I/R was induced as described with minor modifications (Yang et al. 2008).

17 The animals were anesthetized by an intraperitoneal injection of sodium pentobarbital $18(50 \mathrm{mg} / \mathrm{kg})$. After insertion of an endotracheal tube, the animals were placed on positive-pressure ventilation at a tidal volume of $10 \mathrm{~mL} / \mathrm{kg}$ and a rate of 65 strokes $/ \mathrm{min}$. A standard limb lead II electrocardiogram (ECG) was monitored with a cardiograph (BL-

21 420S, Chendu Ltd, China) throughout the experiment. A thoracotomy was performed at 
1 the fourth intercostal space, and the pericardium was opened to expose the heart. The

2 left anterior descending (LAD) coronary artery was ligated 3-4 $\mathrm{mm}$ from its origin by a

$35 / 0$ silk suture with an atraumatic needle. The ends of the ligature were passed through

4 a short length of vinyl tubing. Applying tension to the ligature and pinching off the tube

5 with an arterial clamp resulted in occlusion of the coronary artery and cessation of

6 regional myocardial blood flow. Coronary artery occlusion was maintained for $30 \mathrm{~min}$,

7 after which reperfusion was initiated by withdrawing the vinyl tubing, and the

8 myocardium was reperfused for 60 min. Regional myocardial ischemia was verified by

9 the presence of a zone of cyanosis in the myocardial surface and by a typical elevation

10 of the ST segment in the ECG.

\section{Experimental protocol}

12 Rats were randomly allocated to three groups $(n=10)$ : Sham-operated group, I/R 13 group and MC-treated group. Sham-operated and I/R rats were administered $0.9 \%$ 14 normal saline for 14 days. In the MC-treated group, the rats were administered MC 15 extract $\left(1.98 \mathrm{~g} / \mathrm{kg}\right.$, i.g.) for 14 days. On the $15^{\text {th }}$ day, the rats were subjected to 30 -min 16 LAD coronary artery ligation and 60-min reperfusion. Sham-operated rats were 17 subjected to the same surgical procedure, but the artery was not ligated.

\section{Determination of infarct size}

At the conclusion of the 60-min reperfusion, the coronary artery was reoccluded, and

$202 \mathrm{~mL}$ of Evans Blue (2\%) was injected slowly through a cannulated right carotid artery.

21 The heart was removed from the chest. The left ventricular area was cut into transverse 
1 sections parallel to the atrioventricular groove from the apex to the base. The slices

2 were then incubated with a $1.5 \%$ solution of 2, 3, 5-triphenyltetrazolium chloride (TTC)

3 for $30 \mathrm{~min}$ at $37^{\circ} \mathrm{C}$ to visualize the infarct area. Evans blue-stained areas (the normal

4 left ventricle non-risk region), TTC-stained areas (ischemic but viable tissue), and TTC-

5 negative areas (infarct region) in each slice were cut and weighed. Infarct size is

6 presented as a percentage of the weight of the infarct region to the left ventricle.

\section{Biochemical assays}

$8 \quad$ Blood samples were centrifuged at $3000 \times \mathrm{g}$ for $10 \mathrm{~min}$, and the sera thus obtained

9 were stored at $-80^{\circ} \mathrm{C}$ until assay. Superoxide dismutase (SOD), malondialdehyde

10 (MDA), glutathione (GSH), lactate dehydrogenase (LDH) and creatine kinase (CK)

11 levels were measured with commercial kits according to the manufacturer's directions

12 (Nanjing Jiancheng Institute of Biotechnology, Nanjing, China).

13

\section{Histopathological examination}

At the end of the experiment, the infarct region of the myocardial tissue was immediately fixed in $10 \%$ buffered neutral formalin solution and embedded in paraffin. After processing, wax-embedded sections were cut, stained with hematoxylin and eosin, and examined by light microscopy.

\section{Determination of myocardial apoptosis}

To quantify myocardial apoptosis, in situ detection of apoptotic myocytes was performed by using terminal deoxynucleotidyl uridine triphosphate transferase nick-end 
1 labeling (TUNEL) assay as described previously (Ha et al. 2008). Cardiomyocytes from

2 at least five slides per block were randomly selected and evaluated using

3 immunohistochemistry to determine the number and percentage of cells exhibiting

4 positive apoptotic staining under a light microscope. For each slide, five fields were

5 randomly chosen and counted by image-pro plus 6.0. The apoptotic index (AI) is

6 expressed as the number of TUNEL-positive myocytes/the total number of myocytes.

7 Real-time polymerase chain reaction (PCR)

8 Total RNA was isolated using Simply P Total RNA Extraction kit (Bioflux, Japan)

9 according to the manufacturer's protocol. Single-strand cDNA was synthesized from 2 $10 \mu \mathrm{g}$ of RNA by reverse transcription RevertAid ${ }^{\mathrm{TM}}$ First strand cDNA Synthesis Kit 11 (Thermo, Maryland, USA). Real-time PCR was performed in an iCycler machine (Bio12 Rad, California, USA) using SsoFast ${ }^{\mathrm{TM}}$ EvaGreen Supermix (Bio-Rad, Hercules, USA). 13 All PCR primer sequences (Invitrogen Life Technologies, Shang Hai, China) are listed in

14 Table 1. In all cases, melting point analysis revealed amplification of a single product. 15 Glyceraldehyde-3-phosphate dehydrogenase (GAPDH) was used as an internal 16 standard to monitor loading variations. The PCR products were separated on agarose 17 gel and visualized with the Gel Doc-IT ${ }^{\mathrm{TM}}$ imaging system (Ultra-Violet Products Ltd., 18 California, USA) in the presence of GeIRed ${ }^{\top M}$ (Biotium, USA).

19

\section{Statistical analysis}

Values are presented as the means \pm S.E.M. Statistical analysis was performed with one-way analysis of variance followed by Bonferroni correction for post hoc t-test (SPSS 
1 Version 17.0 [SPSS, Inc., Chicago, USA]). A $P$ value of less than 0.05 was considered

2 statistically significant.

3 Results

$4 \quad$ MC attenuated myocardial infarct size

5 As shown in Figure 1 , the infarct size in myocardial $\mathrm{I} / \mathrm{R}$ rats was increased 6 significantly compared with the sham group $(P<0.05)$. In comparison with the I/R group, 7 the infarct size in the MC-treated rats was reduced by $32.1 \%(P<0.05)$.

\section{MC inhibited I/R-induced oxidative damage}

9 The activities of SOD and GSH were suppressed (20.5\% and $54.6 \%$, respectively) 10 in $\mathrm{I} / \mathrm{R}$ rats relative to the sham group $(P<0.05)$. Furthermore, $\mathrm{I} / \mathrm{R}$ caused an obvious 11 increase in lipid peroxide by $31.7 \%(P<0.05)$. In comparison with the $\mathrm{I} / \mathrm{R}$ group, the

12 activities of SOD and GSH were significantly increased ( $16.2 \%$ and $73.1 \%$, respectively) 13 in MC-treated rats $(P<0.01)$, and MC significantly reduced MDA levels by $13.4 \%(P<$ $140.05)$ (Figure 2).

\section{MC attenuated LDH and CK activities}

I/R rats exhibited a remarkable elevation of LDH and CK activities in comparison

17 with the sham group $(P<0.01$ and $P<0.05$, respectively). Relative to the $\mathrm{I} / \mathrm{R}$ group, $\mathrm{MC}$ decreased the activity of CK by $30.1 \%(P<0.05)$. The LDH level in MC-treated rats was

19 slightly reduced, although no statistical significance was noted (Figure 3). 
MC protected histopathological damage

Figure 4 presents representative photomicrographs of each group. The myocardium in the sham group exhibited normal myocardial architecture. However, rat hearts subjected to $\mathrm{I} / \mathrm{R}$ exhibited myocardial membrane damage, infiltration of inflammatory cells and confluent areas of myonecrosis relative to those in the sham group. MCtreated rats exhibited marked improvement in the degree of myonecrosis, edema and infiltration of inflammatory cells in comparison with the I/R group.

\section{MC attenuated I/R-induced myocardial apoptosis}

(1)

The TUNEL assay was utilized to analyze the influence of $M C$ on the induction of myocardial apoptosis. As illustrated in Figure 5, few apoptotic cells were observed in the hearts of sham-treated rats. I/R resulted in a significant increase in TUNEL-positive nuclei (Al: $35.4 \pm 3.0 \%, P<0.05$ vs. sham group). MC caused a significant reduction in the Al compared with the I/R group (Al: $19.3 \pm 1.6 \%, P<0.05 \mathrm{vs.} \mathrm{I/R} \mathrm{group).}$

\section{$M C$ increased the ratio of $\mathrm{B}$-cell leukemia-2/Bcl-2-associated $\mathrm{X}$ protein (Bcl-2/Bax)} and decreased inducible nitric oxide synthase (iNOS) mRNA expression

Figure 6 presents the PCR result demonstrating the mRNA expression of $\mathrm{Bcl}-2$, Bax and iNOS. A significant decrease was observed in the expression of Bcl-2 mRNA $(P<0.01)$, and increased expression of Bax $(P<0.05)$ and iNOS mRNA $(P<0.01)$ was observed in the I/R group relative to sham-treated rats. Compared with the I/R group, MC increased the mRNA expression of Bcl-2 by 1.05 -fold $(P<0.05)$. Although the mRNA expression of Bax was slightly reduced, the ratio of Bcl-2/Bax was increased by 
1 1.3-fold. Treatment of MC also caused a remarkable decrease in the mRNA expression

2 of iNOS by $33.9 \%(P<0.05)$.

3 Discussion

4 The present study was performed to investigate the effects of MC on myocardial I/R 5 and to explore its possible mechanisms. The administration of MC for 14 days 6 significantly reduced the infarct size of $I / R$ rat hearts relative to the sham group. Our 7 studies also demonstrated the cardioprotective effects of $M C$, as evidenced by marked 8 improvement in the histopathological examination.

9 Oxidative stress has emerged as an important mechanism of myocardial I/R injury. Our findings of depressed SOD and GSH activities and increased MDA level in I/R rats 11 were consistent with earlier reports (Liao et al. 2011; Zhao et al. 2012). SOD is an 12 antioxidant enzyme that catalyzes the conversion of $\mathrm{O}_{2}$ into $\mathrm{H}_{2} \mathrm{O}_{2}$ and $\mathrm{O}_{2}$. GSH is another key factor involved in the detoxification of electrophonic metabolites and 14 reactive oxygen intermediates. The decreased activities of SOD and GSH indicate the failure of the antioxidant defense mechanisms to prevent the formation of excessive free radicals (Rodrigo et al. 2013). The excessive free radicals are capable of inducing lipid peroxidation of membranes, altering membrane integrity and increasing membrane fluidity and permeability (Chang et al. 2013). Leakage of a variety of intracellular enzymes, including CK and LDH, from myocardial tissues into blood is an indicator of acute myocardial infarction (Amani et al. 2013). In the present study, the administration

21 of MC restored SOD and GSH activities and inhibited the formation of lipid peroxide 22 products during I/R. The above results indicated that $M C$ effectively restored the anti- 
1 oxidative defense system, thereby protecting the myocardium from $\mathrm{l} / \mathrm{R}$ injury.

2 Accordingly, I/R-induced elevation of blood CK and LDH was blunted when rats were 3 pretreated with MC.

$4 \quad$ In the present study, MC reduced myocardial apoptosis, as indicated by a reduction 5 in TUNEL-positive myocytes. Apoptosis plays a crucial role in the development of 6 myocardial infarction and I/R (Ale et al. 2013; Liou et al. 2011; Loan Le et al. 2012), 7 which are related to the pathogenesis of heart failure. Many genes have been reported 8 to be linked to the regulation of programmed cell death under physiological and 9 pathological conditions, and the Bcl-2 and Bax genes are suggested to play a central 10 role (Wang et al. 2013). Since Oltvai et al. provided the first demonstration that the 11 ratio of Bcl-2/Bax determines survival or death following an apoptotic stimulus in 1993 12 (Oltvai et al. 1993), the role of Bcl-2 and Bax in disease has been extensively studied. 13 Over expression of $\mathrm{Bcl}-2$ in a line of transgenic mice renders the heart more resistant 14 to apoptosis and I/R injury (Chen et al. 2001). Bax-knockout mice exhibit superior 15 tolerance to I/R damage (Ben-Ari et al. 2007; Hochhauser et al. 2003). Bcl-2 and Bax 16 have emerged as potential drug discovery targets for I/R injury (Reed 2006). In the 17 present study, MC pretreatment increased the mRNA expression of the anti-apoptotic gene $\mathrm{Bcl}-2$ while decreasing the mRNA expression of the proapoptotic gene Bax slightly. We suggest that MC might inhibit cardiomyocyte apoptosis by increasing the 20 ratio of $\mathrm{Bcl}-2 / \mathrm{Bax}$.

We also tested the mRNA expression of iNOS. The iNOS enzyme is responsible for 22 the synthesis of nitric oxide (NO), a free radical that plays a pivotal role in physiology 
1 and pathology in various systems (Chen et al. 2007). iNOS is expressed and activated

2 by stimulating factors during pathologic events (Kanko et al. 2006). NO is a double-

3 edged sword in myocardial I/R and heart failure. Basal levels of NO production from

4 endothelial nitric oxide synthase (eNOS) protect cardiomyocytes from apoptosis,

5 whereas over-production of NO by iNOS promotes apoptosis (Razavi et al. 2005).

6 Excess $\mathrm{NO}$ and the co-existence of ROS with NO are injurious to cardiac cells (Otani

7 2009). In the present study, significantly increased expression of iNOS mRNA was

8 observed in the I/R group, whereas MC caused a remarkable decrease in the mRNA

9 expression of iNOS. We suggest that MC may reduce the excess NO produced during

10 the process of $I / R$ and may exert protective effects. The down-regulation of iNOS

11 reported here is similar to earlier studies demonstrating that MC inhibited iNOS

12 expression in other pathologies, such as lipopolysaccharide- and recombinant

13 interferon-gamma-induced inflammation (Chun et al. 2007; Chung et al. 2007).

14 In conclusion, the reduction of infarct size and the morphological results

15 demonstrated that $M C$ was effective in the protection of I/R injury. The cardioprotective

16 effect of MC was associated with its anti-oxidative and anti-apoptotic activities. MC

17 might have a therapeutic role in improving clinical outcomes in patients with coronary 18 heart disease.

\section{Acknowledgements}

This project was financially supported by a general grant from the National Natural

21 Science Foundation of China (No. 81073046). 


\section{Conflict of interest}

The authors declare no conflict of interest.

\section{References}

Ale, A., Siebenhaar, F., Kosanke, K., Aichler, M., Radrich, K., Heydrich, S., et al. 2013. Cardioprotective C-kit(+) bone marrow cells attenuate apoptosis after acute myocardial infarction in mice - in-vivo assessment with fluorescence molecular imaging. Theranostics, 3(11): 903-913. doi: 10.7150/thno.5938. PMID: 24312159.

Amani, M., Jeddi, S., Ahmadiasl, N., Usefzade, N., and Zaman, J. 2013. Effect of HEMADO on Level of CK-MB and LDH Enzymes after Ischemia/Reperfusion Injury in Isolated Rat Heart. Bioimpacts, 3(2): 101-104. doi: 10.5681/bi.2013.003. PMID: 23878794.

Ben-Ari, Z., Pappo, O., Cheporko, Y., Yasovich, N., Offen, D., Shainberg, A., et al. 2007. Bax ablation protects against hepatic ischemia/reperfusion injury in transgenic mice. Liver Transpl. 13(8): 1181-1188. doi: 10.1002/It.21221. PMID: 17663392.

Chang, G., Zhang, P., Ye, L., Lu, K., Wang, Y., Duan, Q., et al. 2013. Protective effects of sitagliptin on myocardial injury and cardiac function in an ischemia/reperfusion rat model. Eur. J. Pharmacol. 718(1-3): 105-113. doi: 10.1016/j.ejphar.2013.09.007. PMID: 24041927.

Chen, G., Zhang, L., and Yang, P. 2005. Determination of three bioactive constituents in moutan cortex by capillary electrophoresis with electrochemical detection. Anal. Sci. 21(10): 1161-1165. PMID: 16270571. 
Chen, H.I., Chang, H.R., Wu, C.Y., Kao, S.J., Wang, D., Hsieh, N.K., et al. 2007. Nitric oxide in the cardiovascular and pulmonary circulation--a brief review of literatures and historical landmarks. Chin. J. Physiol. 50(2): 43-50. PMID: 17608140.

Chen, Z., Chua, C.C., Ho, Y.S., Hamdy, R.C., and Chua, B.H. 2001. Overexpression of $\mathrm{Bcl}-2$ attenuates apoptosis and protects against myocardial I/R injury in transgenic mice. Am. J. Physiol. 280(5): H2313-2320. PMID: 11299236.

Chun, S.C., Jee, S.Y., Lee, S.G., Park, S.J., Lee, J.R., and Kim, S.C. 2007. Antiinflammatory activity of the methanol extract of moutan cortex in LPS-activated Raw264.7 cells. Evid. Based Complement. Alternat. Med. 4(3): 327-333. doi: 10.1093/ecam/nel093. PMID: 17965763.

Chung, H.S., Kang, M., Cho, C., Parvez, S., Park, C.H., Kim, D., et al. 2007. Inhibition of nitric oxide and tumor necrosis factor-alpha by moutan cortex in activated mouse peritoneal macrophages. Biol. Pharm. Bull. 30(5): 912-916. PMID: 17473434.

Dobsak, P., Siegelova, J., Eicher, J.C., Jancik, J., Svacinova, H., Vasku, J., et al. 2003. Melatonin protects against ischemia-reperfusion injury and inhibits apoptosis in isolated working rat heart. Pathophysiology, 9(3): 179-187. PMID: 14567933.

Dominguez-Rodriguez, A., and Abreu-Gonzalez, P. 2010. Myocardial ischemiareperfusion injury: Possible role of melatonin. World J. Cardiol. 2(8): 233-236. doi: 10.4330/wjc.v2.i8.233. PMID: 21160589.

Dominguez-Rodriguez, A., Abreu-Gonzalez, P., and Reiter, R.J. 2014. Cardioprotection and pharmacological therapies in acute myocardial infarction: Challenges in the 
current era. World J. Cardiol. 6(3): 100-106. doi: 10.4330/wjc.v6.i3.100. PMID: 24669291.

Fu, P.K., Yang, C.Y., Tsai, T.H., and Hsieh, C.L. 2012. Moutan cortex radicis improves lipopolysaccharide-induced acute lung injury in rats through anti-inflammation. Phytomedicine, 19(13): 1206-1215. doi: 10.1016/j.phymed.2012.07.013. PMID: 22921747.

Gershlick, A.H., Banning, A.P., Myat, A., Verheugt, F.W., and Gersh, B.J. 2013. Reperfusion therapy for STEMI: is there still a role for thrombolysis in the era of primary percutaneous coronary intervention? Lancet, 382(9892): 624-632. doi: 10.1016/S0140-6736(13)61454-3. PMID: 23953386.

Ha, T., Hua, F., Liu, X., Ma, J., McMullen, J.R., Shioi, T., et al. 2008. Lipopolysaccharide-induced myocardial protection against ischaemia/reperfusion injury is mediated through a PI3K/Akt-dependent mechanism. Cardiovasc. Res. 78(3): 546-553. doi: 10.1093/cvr/cvn037. PMID: 18267957.

Hochhauser, E., Kivity, S., Offen, D., Maulik, N., Otani, H., Barhum, Y., et al. 2003. Bax ablation protects against myocardial ischemia-reperfusion injury in transgenic mice. Am. J. Physiol. 284(6): H2351-2359. doi: 10.1152/ajpheart.00783.2002. PMID: 12742833.

Inafuku, H., Kuniyoshi, Y., Yamashiro, S., Arakaki, K., Nagano, T., Morishima, Y., et al. 2013. Determination of oxidative stress and cardiac dysfunction after ischemia/reperfusion injury in isolated rat hearts. Ann. Thorac. Cardiovasc. Surg. 19(3): 186-194. PMID: 22971810. 
1 Jiang, S., Nakano, Y., Yatsuzuka, R., Ono, R., and Kamei, C. 2007. Inhibitory effects of Moutan cortex on immediate allergic reactions. Biol. Pharm. Bull. 30(9): 1707-1710. PMID: 17827725.

Kanko, M., Ozden, M., Maral, H., and Acil, C. 2006. Effect of clopidogrel on nitric oxide levels in an ischemia reperfusion model. J. Cardiovasc. Pharmacol. 48(1): 797-801. doi: 10.1097/01.fjc.0000211795.45281.9d. PMID: 16891907.

Liao, X., Wang, L., Yang, C., He, J., Wang, X., Guo, R., et al. 2011. Cyclooxygenase mediates cardioprotection of angiotensin-(1-7) against ischemia/reperfusioninduced injury through the inhibition of oxidative stress. Mol. Med. Rep. 4(6): 11451150. doi: 10.3892/mmr.2011.570. PMID: 21874245.

Liou, S.F., Ke, H.J., Hsu, J.H., Liang, J.C., Lin, H.H., Chen, I.J., et al. 2011. San-HuangXie-Xin-Tang Prevents Rat Hearts from Ischemia/Reperfusion-Induced Apoptosis through eNOS and MAPK Pathways. Evid. Based Complement. Alternat. Med. 2011: 915051 . doi: 10.1093/ecam/neq061. PMID: 21785641.

Liu, K.Y., Hu, S., Chan, B.C., Wat, E.C., Lau, C.B., Hon, K.L., et al. 2013. Antiinflammatory and anti-allergic activities of Pentaherb formula, Moutan Cortex (Danpi) and gallic acid. Molecules, 18(3): 2483-2500. doi: 10.3390/molecules18032483. PMID: 23439564.

Loan Le, T.Y., Mardini, M., Howell, V.M., Funder, J.W., Ashton, A.W., and Mihailidou, A.S. 2012. Low-dose spironolactone prevents apoptosis repressor with caspase recruitment domain degradation during myocardial infarction. Hypertension, 59(6): 1164-1169. doi: 10.1161/HYPERTENSIONAHA.111.190488. PMID: 22508833. 
1 Mohanty, I., Arya, D.S., and Gupta, S.K. 2006. Effect of Curcuma longa and Ocimum sanctum on myocardial apoptosis in experimentally induced myocardial ischemicreperfusion injury. BMC Complement. Altern. Med. 6: 3. doi: 10.1186/1472-6882-63. PMID: 16504000.

Oltvai, Z.N., Milliman, C.L., and Korsmeyer, S.J. 1993. Bcl-2 heterodimerizes in vivo with a conserved homolog, Bax, that accelerates programmed cell death. Cell, 74(4): 609-619. PMID: 8358790.

Otani, H. 2009. The role of nitric oxide in myocardial repair and remodeling. Antioxid. Redox. Signal. 11(8): 1913-1928. doi: 10.1089/ARS.2009.2453. PMID: 19203224.

Pagliaro, P., and Penna, C. 2015. Redox signalling and cardioprotection: translatability and mechanism. Br. J. Pharmacol. 172(8):1974-1995. doi: 10.1111/bph.12975. PMID: 25303224.

Razavi, H.M., Hamilton, J.A., and Feng, Q. 2005. Modulation of apoptosis by nitric oxide: implications in myocardial ischemia and heart failure. Pharmacol. Ther. 106(2): 147-162. doi: 10.1016/j.pharmthera.2004.11.006. PMID: 15866317.

Reed, J.C. 2006. Proapoptotic multidomain Bcl-2/Bax-family proteins: mechanisms, physiological roles, and therapeutic opportunities. Cell Death Differ. 13(8): 13781386. doi: 10.1038/sj.cdd.4401975. PMID: 16729025.

Rho, S., Chung, H.S., Kang, M., Lee, E., Cho, C., Kim, H., et al. 2005. Inhibition of production of reactive oxygen species and gene expression profile by treatment of 
ethanol extract of Moutan Cortex Radicis in oxidative stressed PC12 cells. Biol. Pharm. Bull. 28(4): 661-666. PMID: 15802806.

Rodrigo, R., Libuy, M., Feliu, F., and Hasson, D. 2013. Oxidative stress-related biomarkers in essential hypertension and ischemia-reperfusion myocardial damage. Dis. Markers, 35(6): 773-790. doi: 10.1155/2013/974358. PMID: 24347798.

Venugopal, J.R., Prabhakaran, M.P., Mukherjee, S., Ravichandran, R., Dan, K., and Ramakrishna, S. 2012. Biomaterial strategies for alleviation of myocardial infarction. J. R. Soc. Interface, 9(66): 1-19. doi: 10.1098/rsif.2011.0301. PMID: 21900319.

Wang, Y., Li, X., Wang, X., Lau, W., Wang, Y., Xing, Y., et al. 2013. Ginsenoside Rd attenuates myocardial ischemia/reperfusion injury via Akt/GSK-3beta signaling and inhibition of the mitochondria-dependent apoptotic pathway. PLoS One, 8(8): e70956. doi: 10.1371/journal.pone.0070956. PMID: 23976968.

Wu, M., and Gu, Z. 2009. Screening of bioactive compounds from moutan cortex and their anti-inflammatory activities in rat synoviocytes. Evid Based Complement. Alternat. Med. 6(1): 57-63. doi: 10.1093/ecam/nem066. PMID: 18955220.

Yang, J., Yang, J., Ding, J.W., Chen, L.H., Wang, Y.L., Li, S., et al. 2008. Sequential expression of TLR4 and its effects on the myocardium of rats with myocardial ischemia-reperfusion injury. Inflammation, 31(5): 304-312. doi: 10.1007/s10753008-9079-x. PMID: 18677579.

Yun, C.S., Choi, Y.G., Jeong, M.Y., Lee, J.H., and Lim, S. 2013. Moutan Cortex Radicis inhibits inflammatory changes of gene expression in lipopolysaccharide-stimulated 

gingival fibroblasts. J. Nat. Med. 67(3): 576-589. doi: 10.1007/s11418-012-0714-3. PMID: 23086154.

3 Zhang, M., Feng, L., Gu, J., Ma, L., Qin, D., Wu, C., et al. 2014. The attenuation of Moutan Cortex on oxidative stress for renal injury in AGEs-induced mesangial cell

5 dysfunction and streptozotocin-induced diabetic nephropathy rats. Oxid. Med. Cell Longev. 2014: 463815. doi: 10.1155/2014/463815. PMID: 24876912.

7 Zhao, L., Peng, D.Q., Zhang, J., Song, J.Q., Teng, X., Yu, Y.R., et al. 2012.

8 Extracellular signal-regulated kinase 1/2 activation is involved in intermedin1-53 attenuating myocardial oxidative stress injury induced by ischemia/reperfusion. Peptides, 33(2): 329-335. doi: 10.1016/j.peptides.2011.12.016. PMID: 22244813. 
1 Table 1. Primer sequences of B-cell leukemia-2 (Bcl-2), Bcl-2-associated X protein 2 (Bax), inducible nitric oxide synthase (iNOS) and glyceraldehyde-3-phosphate 3 dehydrogenase (GAPDH).

\section{Gene name Primer sequence (5'-3')}

\begin{tabular}{ll}
\hline Bax & Forward: TGCAGAGGATGATTGCTGAC
\end{tabular}

Reverse: GATCAGCTCGGGCACTTTAG

Bcl-2

Forward: ATACCTGGGCCACAAGTGAG

Reverse: TGATTTGACCATTTGCCTGA

iNOS

Forward: GGAGAGATTTTTCACGACACCC

Reverse: CCATGCATAATTTGGACTTGCA

GAPDH Forward: CGCTAACATCAAATGGGGTG

Reverse: TTGCTGACAATCTTGAGGGAG 


\section{Figure legends}

2 Fig. 1. Pretreatment with Moutan Cortex (MC) extract decreased the infarct size in

3 ischemia/reperfusion $(\mathrm{I} / \mathrm{R})$ rats. The data are the means \pm S.E.M. $(n=10) .{ }^{*} P<0.05$ vs.

4 sham group; ${ }^{\#} P<0.05$ vs. I/R group.

5 Fig. 2. The activities of superoxide dismutase (SOD) and glutathione (GSH) were

6 increased by pretreatment with Moutan Cortex (MC) extract in ischemia/reperfusion (I/R)

7 rats, whereas the level of malondialdehyde (MDA) was reduced by MC. The data are

8 the means \pm S.E.M. $(\mathrm{n}=10) .{ }^{*} P<0.05$ vs. Sham group; ${ }^{\# \#} P<0.01$ vs. I/R group.

9 Fig. 3. Moutan Cortex (MC) extract attenuated the activity of lactate dehydrogenase

$10(\mathrm{LDH})$ and creatine kinase $(\mathrm{CK})$ in ischemia/reperfusion (I/R) rats. The data are the

11 means \pm S.E.M. $(\mathrm{n}=10) .{ }^{*} P<0.05$ vs. sham group; ${ }^{* *} P<0.01$ vs. sham group; ${ }^{\# \#} P<$

120.01 vs. I/R group.

13 Fig. 4. Moutan Cortex (MC) extract protected against histopathological damage in 14 ischemia/reperfusion (I/R) rat hearts stained by hematoxylin and eosin. (A) Sham group, 15 (B) I/R group, (C) MC group.

Fig. 5. Moutan Cortex (MC) extract attenuated myocardial apoptosis in

17 ischemia/reperfusion (I/R) rats. Representative photomicrographs of heart tissues of the 18 sham group (A), I/R group (B) and MC group (C). The apoptotic index (AI) is expressed 19 as a percentage of TUNEL-positive myocytes in tissue sections. The data are the 20 means \pm S.E.M. $(\mathrm{n}=5) .{ }^{* *} P<0.01$ vs.sham group; ${ }^{\# \#} P<0.01$ vs. I/R group. 
1 Fig. 6. Moutan Cortex (MC) extract increased the mRNA expression of B-cell leukemia22 (Bcl-2) and inducible nitric oxide synthase (iNOS) while decreasing Bcl-2-associated X 3 protein (Bax) mRNA expression slightly in ischemia/reperfusion (I/R) rats. 4 Glyceraldehyde-3-phosphate dehydrogenase (GAPDH) was used as an internal 5 standard. The data are the means \pm S.E.M. $(n=5) .{ }^{*} P<0.05$ vs. Sham group; ${ }^{* *} P<0.01$ 6 vs. Sham group; ${ }^{\#} P<0.05$ vs. I/R group. 


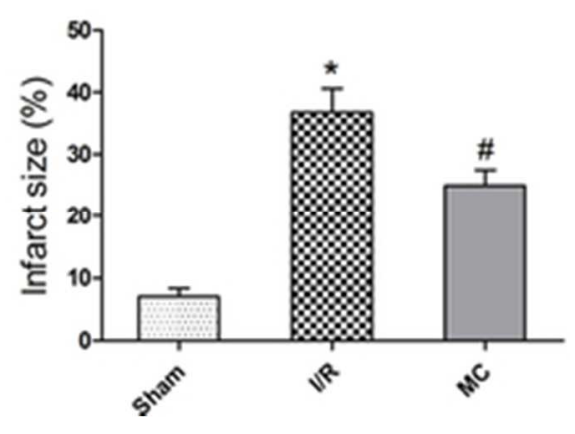

Fig. 1. Pretreatment with Moutan Cortex (MC) extract decreased the infarct size in ischemia/reperfusion $(I / R)$ rats. The data are the means \pm S.E.M. $(n=10)$. ${ }^{P}<<0.05$ vs. sham group; \#P $<0.05$ vs. I/R group. $9 \times 6 \mathrm{~mm}(600 \times 600 \mathrm{DPI})$ 

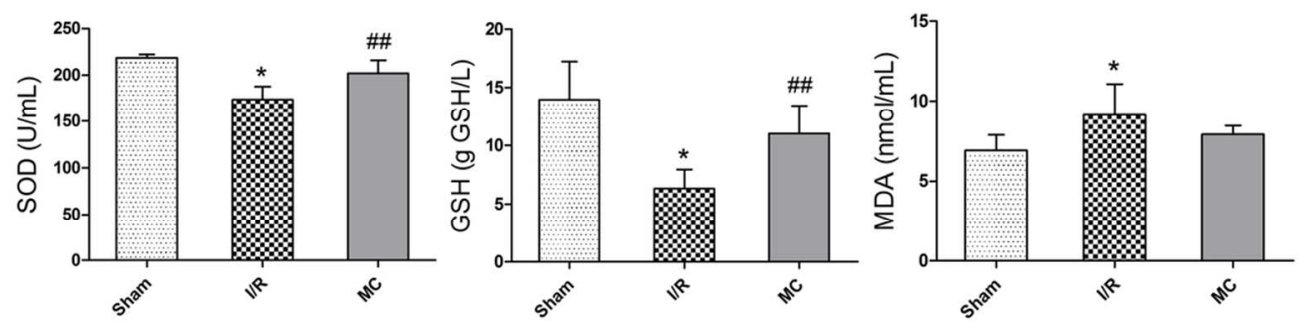

Fig. 2. The activities of superoxide dismutase (SOD) and glutathione (GSH) were increased by pretreatment with Moutan Cortex (MC) extract in ischemia/reperfusion (I/R) rats, whereas the level of malondialdehyde (MDA) was reduced by MC. The data are the means \pm S.E.M. $(n=10)$. ${ }^{*} P<0.05$ vs. Sham group; \#\#P< 0.01 vs. I/R group.

$48 \times 12 \mathrm{~mm}(600 \times 600 \mathrm{DPI})$ 

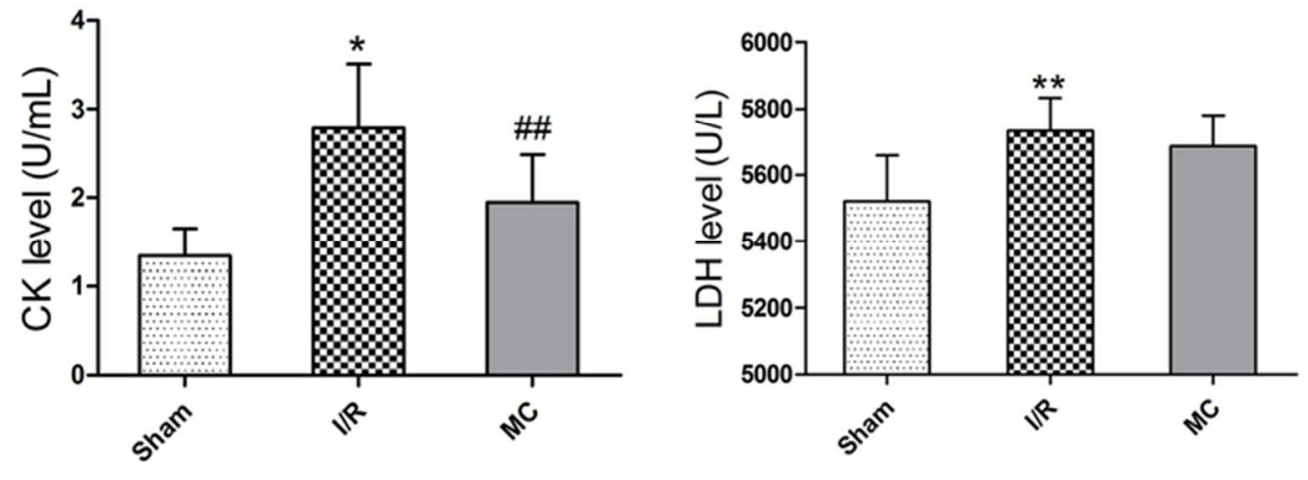

Fig. 3. Moutan Cortex (MC) extract attenuated the activity of lactate dehydrogenase (LDH) and creatine kinase $(\mathrm{CK})$ in ischemia/reperfusion (I/R) rats. The data are the means \pm S.E.M. $(n=10) . * P<0.05$ vs. sham group; $* * P<0.01$ vs. sham group; \#\#P $<0.01$ vs. I/R group.

$30 \times 11 \mathrm{~mm}(600 \times 600 \mathrm{DPI})$ 

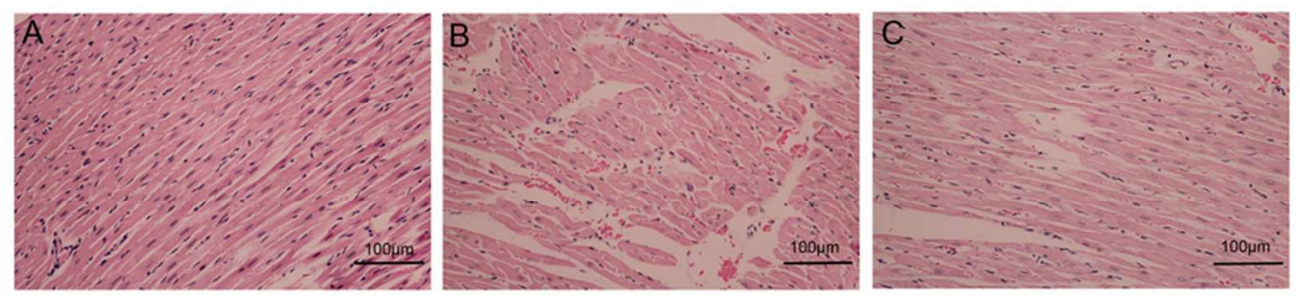

Fig. 4. Moutan Cortex (MC) extract protected against histopathological damage in ischemia/reperfusion (I/R) rat hearts stained by hematoxylin and eosin. (A) Sham group, (B) I/R group, (C) MC group. $38 \times 8 \mathrm{~mm}(600 \times 600 \mathrm{DPI})$ 


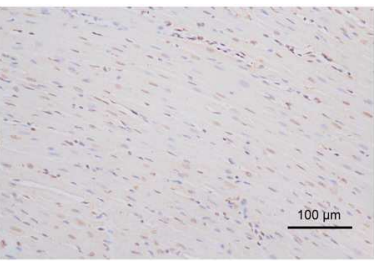

A

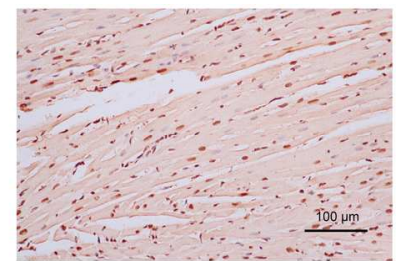

B

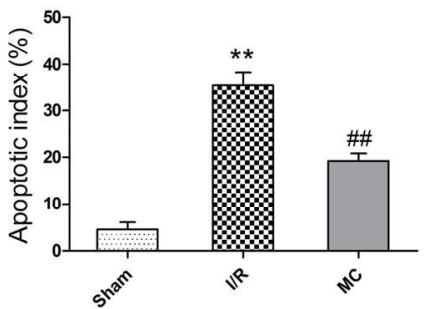

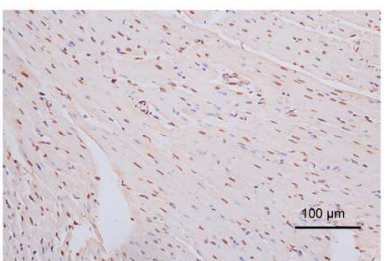

C

Fig. 5. Moutan Cortex (MC) extract attenuated myocardial apoptosis in ischemia/reperfusion (I/R) rats. Representative photomicrographs of heart tissues of the sham group (A), I/R group (B) and MC group (C). The apoptotic index (AI) is expressed as a percentage of TUNEL-positive myocytes in tissue sections. The data are the means \pm S.E.M. $(n=10)$. $* * P<0.01$ vs.sham group; \#\# $<0.01$ vs. I/R group.

$85 \times 43 \mathrm{~mm}(600 \times 600 \mathrm{DPI})$ 


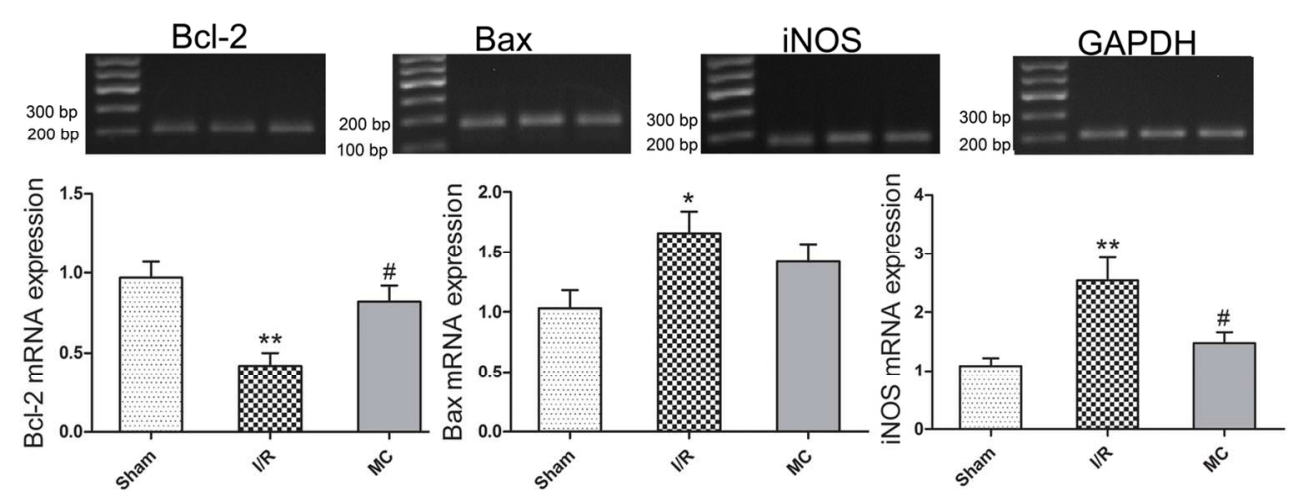

Fig. 6. Moutan Cortex (MC) extract increased the mRNA expression of B-cell leukemia-2 (Bcl-2) and inducible nitric oxide synthase (iNOS) while decreasing Bcl-2-associated X protein (Bax) mRNA expression slightly in ischemia/reperfusion (I/R) rats. Glyceraldehyde-3-phosphate dehydrogenase (GAPDH) was used as an internal standard. The data are the means \pm S.E.M. $(n=5)$. $* P<0.05$ vs. Sham group; $* * P<0.01$ vs. Sham group; \#P $<0.05$ vs. I/R group.

$63 \times 23 \mathrm{~mm}(600 \times 600$ DPI $)$ 